\title{
Standardization of the ultrasound examination of the masseter muscle with size-independent calculation of records
}

\author{
Krzysztof Gawriołek ${ }^{1, A, C-E}$, Tomasz Klatkiewicz ${ }^{1, A-D}$, Agnieszka Przystańska, ${ }^{1, C-E}$, \\ Zofia Maciejewska-Szaniec ${ }^{1, B-D}$, Tomasz Gedrange ${ }^{2, A, C, E, F}$, Agata Czajka-Jakubowska ${ }^{1, A, C, E, F}$ \\ ${ }^{1}$ Department of Temporomandibular Disorders, Division of Prosthodontics, Poznan University of Medical Sciences, Poland \\ ${ }^{2}$ Department of Dental Surgery, Wroclaw Medical University, Poland \\ A - research concept and design; $\mathrm{B}$ - collection and/or assembly of data; $\mathrm{C}$ - data analysis and interpretation; \\ $D$ - writing the article; $E$ - critical revision of the article; $F$ - final approval of the article
}

Address for correspondence

Krzysztof Gawriołek

k.gawriolek@gmail.com

Funding sources

None declared

Conflict of interest

None declared

Received on July 3, 2020

Reviewed on July 28, 2020

Accepted on November 11, 2020

Published online on April 29, 2021

Cite as

Gawriołek K, Klatkiewicz T, Przystańska A, MaciejewskaSzaniec Z, Gedrange I, Czajka-Jakubowska A. Standardization of the ultrasound examination of the masseter muscle with size-independent calculation of records. Adv Clin Exp Med. 2021;30(4):441-447. doi:10.17219/acem/130358

DOI

10.17219/acem/130358

Copyright

Copyright by Author(s)

This is an article distributed under the terms of the

Creative Commons Attribution 3.0 Unported (CC BY 3.0)

(https://creativecommons.org/licenses/by/3.0/)

\begin{abstract}
Background. As ultrasonography provides objective parameters and values, it is a reliable method of examining the structure and dimensions of the masseter muscle. Although the method is well known, there is no standardization in clinical examination and data analysis yet.
\end{abstract}

Objectives. The study aimed to measure masseter muscle thickness in designated areas to establish the most repeatable and clinically applicable method of ultrasound examination, and to assess differences in measurements in designated areas for clinical purposes by devising the size-independent parameter. The size-independent parameter may potentially be more clinically applicable than distance records, which are affected by the size of the subject.

Materials and methods. An ultrasound examination of 124 masseter muscles was performed. Axial examination in 3 horizontal regions (lower, middle and upper) and coronal examination in 2 vertical regions (proximal and distal) was carried out. Masseter muscle thickness was measured in every designated area when relaxed (muscle at rest (RMT)) and with clenched teeth (contracted muscle (CMT)). A morphological independent functional index of thickness difference (FITD) was calculated.

Results. The study revealed very high statistical differences between RMT and CMT ( $p<0.0001)$ in all designated areas but with location variations. Masseter muscle thickness significantly differed depending on the examined area and transducer projection.

Conclusions. The ultrasound study showed that masseter muscle thickness significantly differs depending on the examined area. The authors emphasize the necessity to examine the masseter muscle in specified areas with both coronal and axial projections to achieve objective and repeatable examination. Notable clinical value is assigned to FITD, which is independent from the morphological dimensions of the muscle.

Key words: bruxism, tooth wear, masticatory muscles, masseter ultrasound measurements 


\section{Background}

Ultrasound examination is considered to be useful for functional analysis of the masticatory organ ${ }^{1,2}$ and diagnosis of alterations in the muscles of mastication. ${ }^{3}$ It provides objective parameters and values; it is an effective, accurate, low-cost, and reliable method of examining the structure and dimensions of the masseter muscle. ${ }^{4,5}$ The most often investigated parameter, because of its clinical value and ease of measurement, is masseter muscle thickness. Several ultrasound assessments of masseter muscle thickness in healthy individuals have been published. ${ }^{5-8}$ The morphology of the muscle revealed a large variation in masseter muscle thickness among adult individuals during both relaxed and contracted conditions. ${ }^{5}$ Moreover, significant positive correlations between masseter muscle thickness and some morphological features (i.e., anterior and posterior total face height, vertical jaw length, mandibular ramus height, mandibular length, mandibular inclination) were observed. Also, significant negative correlations with mandibular plane angle and gonial angle were observed., 9 It was also proved that masseter muscle thickness influences the growth of the jaws. ${ }^{11}$

The relation between masseter muscle thickness and clinical findings was proven in numerous studies. Some ultrasound studies were concerned with masseter muscle thickness in relation to dental procedures such as diagnostics and treatment of temporomandibular disorders (TMD), ${ }^{12}$ oral submucous fibrosis, ${ }^{13,14}$ prosthetic treatment,${ }^{15-18}$ and orthodontic treatment, ${ }^{19}$ as well as general clinical findings (i.e., sarcopenia ${ }^{20}$ and osteoporosis ${ }^{21}$ ). It was also believed that muscle thickness is an indicator of muscle function. Its significant correlation to bite force and occlusal tooth contact has been reported. ${ }^{9}$

Although attempts were made to introduce ultrasonography as a routine diagnostic procedure in TMD, the methodological aspects of the examination are unclear and not standardized. ${ }^{22}$ A clear explanation of the examination method or description of the examined region was very rare. Some studies present no relation to examined and measured location, thus not taking under consideration masseter morphological differences in thickness. Others do not specify muscle activity or examine the masseter only at rest or contraction. There is also lack of studies describing differences in clinical ultrasound records at coronal and axial projections. Most current studies present examination using only 1 projection, with no explanation of the advantages of the chosen projection. No paper has presented relationships between the measurements taken and body size.

Our previous review ${ }^{22}$ showed that there are no standards and population normal values. The need for standardization of methods and parameters has been previously reported. ${ }^{7}$ It is believed that various factors can affect the accuracy of measurements of the masseter muscle, with even the use of different conduits a possible cause of inconsistent results. ${ }^{23}$

\section{Objectives}

In this paper, the authors attempted to present a method of standardization of masseter ultrasound examination for clinical needs, with specific regard to the dimensions of the studied muscle and how the examination protocol may affect clinical judgment. Thus, the aim of this ultrasound study was: 1 ) to establish the most repeatable and clinically applicable method of ultrasound examination of the masseter muscle; and 2) to assess differences in measurements in designated areas for clinical purposes by devising the size-independent parameter that potentially may be more clinically applicable than distance records.

\section{Materials and methods}

The study protocol was approved by the local university Bioethics Committee of Poznan University of Medical Sciences. It involved investigation of 80 consecutive asymptomatic participants who volunteered for the study by responding to an invitation (random volunteerns recruited verbally). The participants gave their written informed consent prior to participation in the study. The inclusion criteria were as follows: age 20-30 years, full dentition (except third molars) and no previous TMD treatment. Exclusion criteria were: no referred symptoms of TMDs in the patient's history (e.g., pain from the temporomandibular joint (TMJ) region, movement restriction, muscle weakness, or joint sounds (clicks) (DC/TMD II and III group), radiological and clinical signs of TMJ disc dislocation without reduction or degenerative joint disorders, tooth wear (according to tooth wear index (TWI)), prosthetic reconstructions, or the presence of a systemic disease (e.g., rheumatic or degenerative disease, systemic connective tissue disorders, myasthenia gravis).

All participants were examined with the Diagnostic Criteria for Temporomandibular Disorders (DC/TMD) form. ${ }^{24}$ According to this protocol, 62 consecutive patients qualified for the study (30 men and 32 women). The average age of the patients was 25.4 years. Each individual underwent bilateral ultrasound examination of the masseter muscles. The muscles were examined with the Aloka-Hitachi F37 USG device with $12 \mathrm{MHz}$ linear probe (Aloka-Hitachi, Tokyo, Japan). To achieve the most repeatable examination protocol, the muscle was virtually divided into 3 horizontal regions (lower, middle and upper) and 2 vertical regions (proximal and distal). The axial and coronal ultrasound examinations were carried out in the horizontal and vertical regions, respectively. The thickness of the masseter muscle was measured in every designated area when relaxed (muscle at rest (RMT)), with individuals resting comfortably and associated muscles in a state of minimal contractual activity, and with clenched teeth (contracted muscle (CMT)) in maximal intercuspal position (Fig. 1-3). 


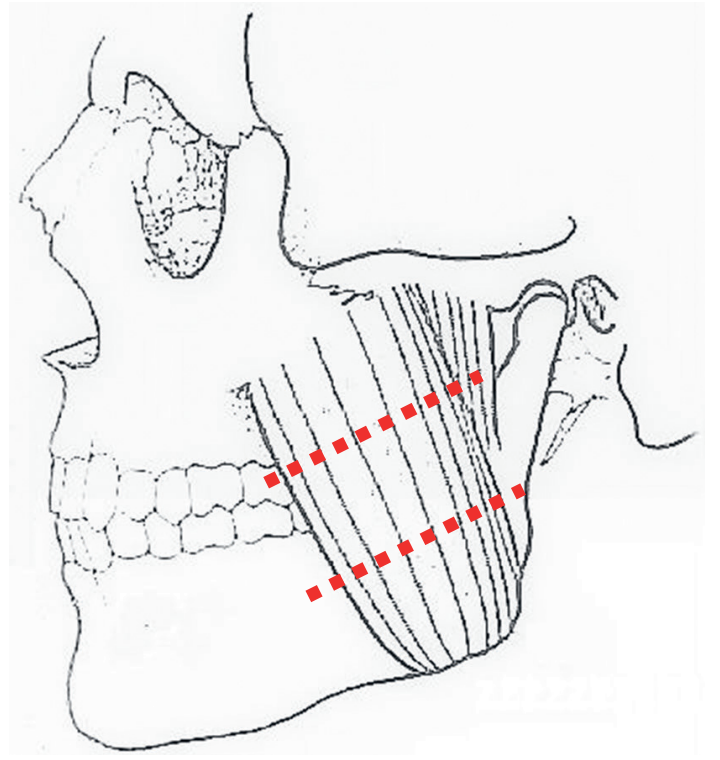

Fig. 1. Schematic diagram of the division into horizontal examination areas (upper, middle and lower), with the example of examination
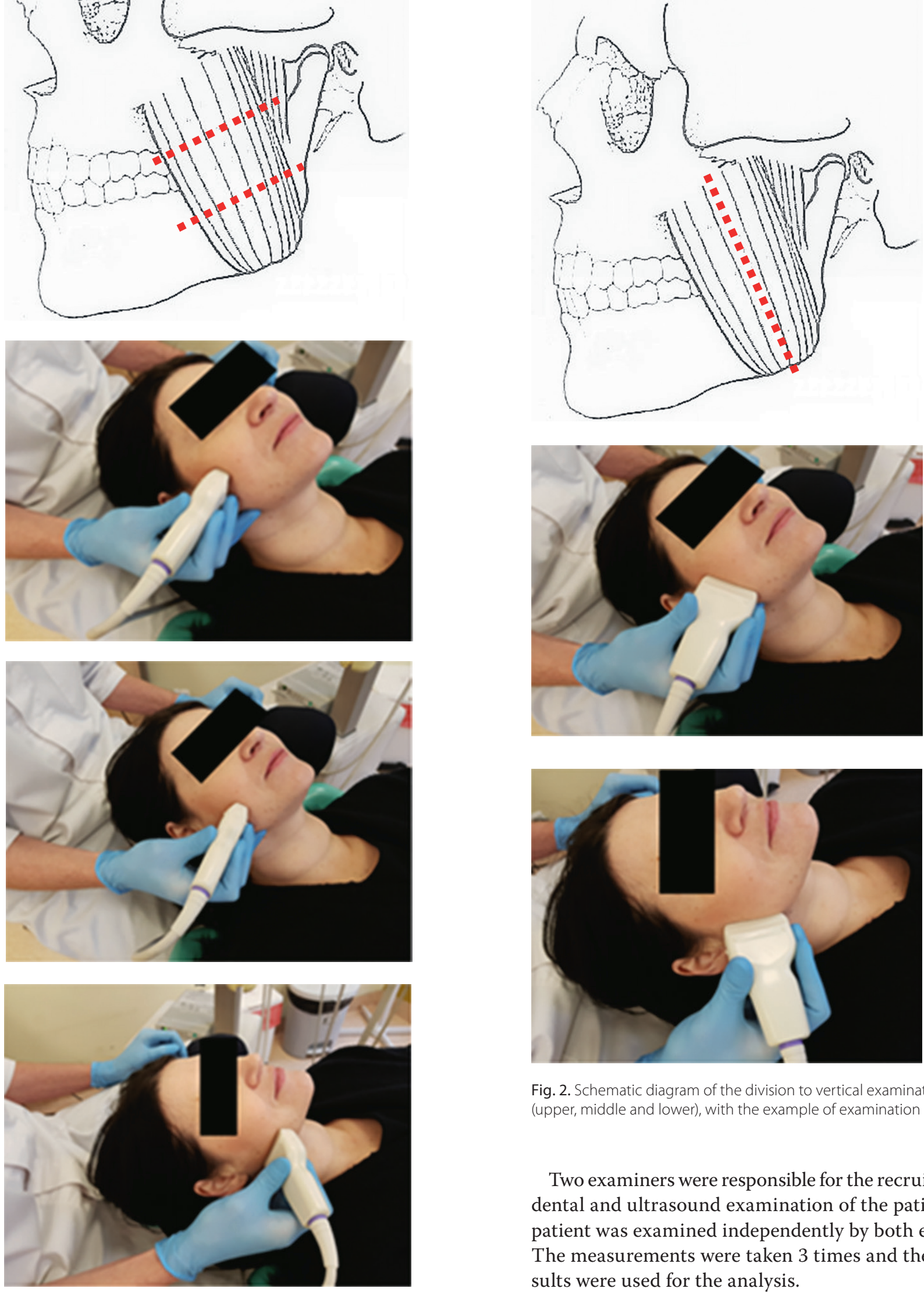

Fig. 2. Schematic diagram of the division to vertical examination areas (upper, middle and lower), with the example of examination

Two examiners were responsible for the recruitment and dental and ultrasound examination of the patients. Each patient was examined independently by both examiners. The measurements were taken 3 times and the mean results were used for the analysis. 

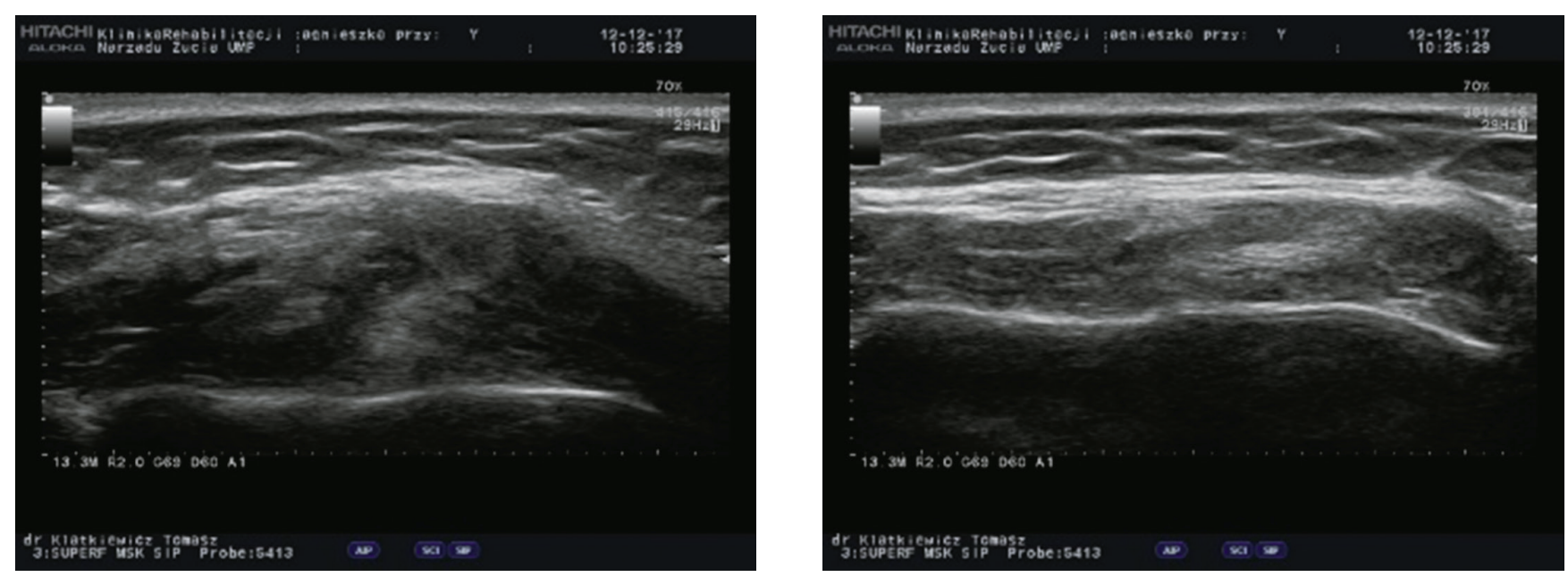

Fig. 3. Ultrasound image at relaxed muscle (RMT) (A) and clenched position (CMT) (B)

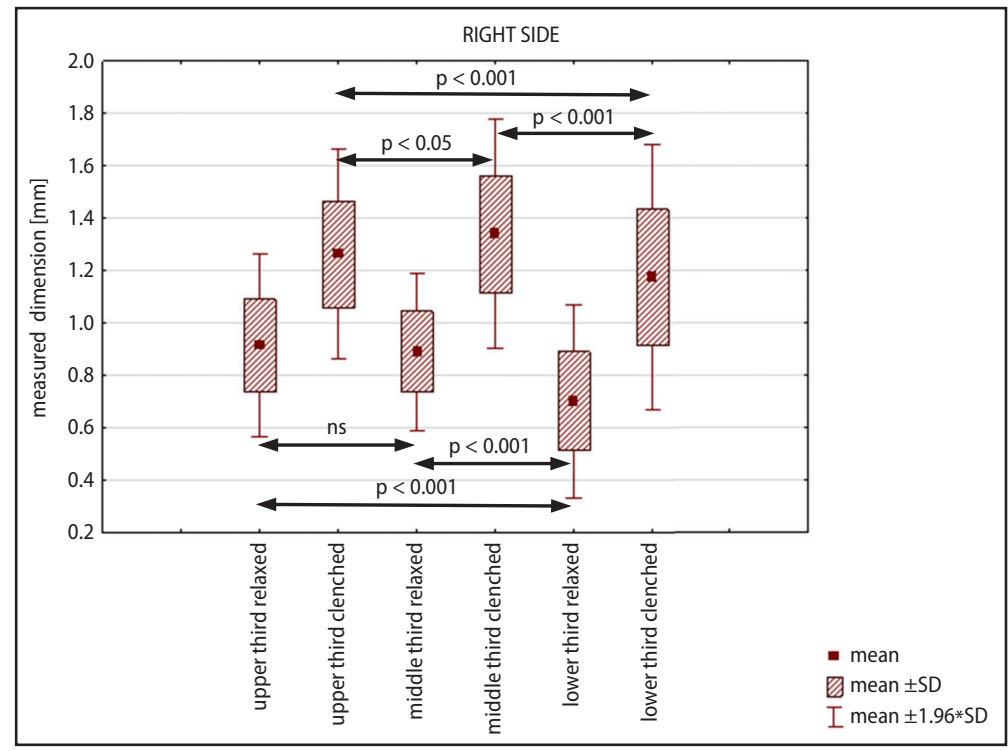

Fig. 4. Statistical imaging of ultrasound measurements at upper, middle and lower areas of masseter at RMT and CMT records, with the p-level on the right side

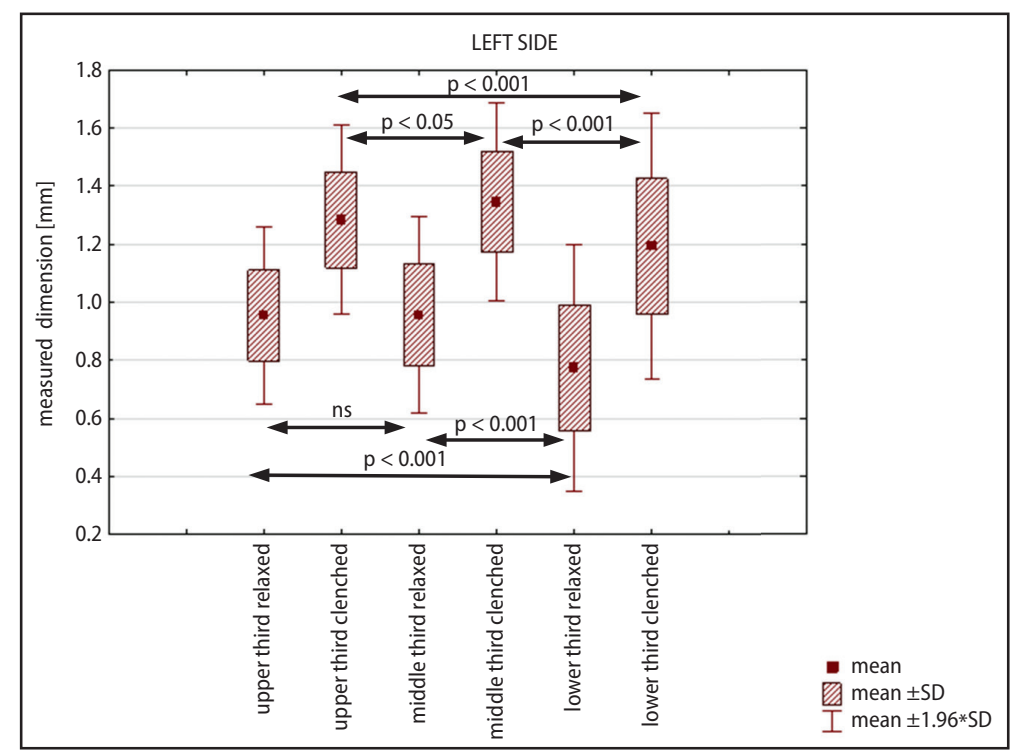

Fig. 5. Statistical imaging of ultrasound measurements at upper, middle and lower areas of masseter at RMT and CMT records, with the p-level on the left side
The authors' original method of calculations included the functional index of thickness difference (FITD). The index was devised to show the change in masseter muscle thickness between contraction and relaxation with no relation to muscle size. It was calculated as the quotient of the difference in values between CMT and RMT.

Data were analyzed using STATISTICA PL v. 12.0 (StatSoft Poland, Kraków, Poland). The analyzed variables expressed in the quantitative scale were presented using the number $(\mathrm{N})$, mean, median, minimum and maximum value, lower and upper quartile, and standard deviation (SD). The Shapiro-Wilk test was used to test the distribution of analyzed variables. Because the analyzed variables significantly differed from the normal distribution, non-parametric tests were applied. The Friedman's test with a post hoc test (Dunn's test) was used to analyze the differences of investigated variables in relation to the muscle thickness examination region (upper, middle or lower), and the Wilcoxon's test was used to test for differences between investigated variables in relation to the masseter muscle thickness examination region (distal or proximal). The analysis was performed for the right and the left side. The assumed significance level was $\mathrm{p}<0.05$.

\section{Results}

The data obtained from every examined area were compared to other areas in the coronal and axial projections. The Friedman's test (axial projection) and Wilcoxon's test (coronal projection) showed statistically significant differences between most of the analyzed areas. No statistically significant differences were found 
Table 1. The masseter muscle thickness [mm] measured in different regions of the muscle $(\mathrm{n}=62)$

\begin{tabular}{|c|c|c|c|c|c|c|c|c|c|c|}
\hline \multirow{2}{*}{$\begin{array}{l}\text { Examination position/ } \\
\text { region/side }\end{array}$} & Mean & Median & Min & Max & SD & Mean & Median & Min & Max & SD \\
\hline & \multicolumn{5}{|c|}{ right side } & \multicolumn{5}{|c|}{ left side } \\
\hline RMT upper third & 0.91 & 0.91 & 0.56 & 1.36 & 0.18 & 0.95 & 0.94 & 0.62 & 1.34 & 0.16 \\
\hline RMT middle third & 0.89 & 0.89 & 0.57 & 1.22 & 0.15 & 0.96 & 0.95 & 0.57 & 1.52 & 0.17 \\
\hline RMT lower third & 0.70 & 0.67 & 0.44 & 1.18 & 0.19 & 0.78 & 0.75 & 0.42 & 1.75 & 0.22 \\
\hline RMT proximal & 1.00 & 1.01 & 0.62 & 1.30 & 0.16 & 1.03 & 1.03 & 0.70 & 1.35 & 0.16 \\
\hline RMT distal & 0.96 & 0.95 & 0.57 & 1.31 & 0.18 & 0.97 & 0.95 & 0.57 & 1.28 & 0.17 \\
\hline CMT upper third & 1.26 & 1.25 & 0.78 & 1.68 & 0.20 & 1.28 & 1.29 & 0.90 & 1.64 & 0.16 \\
\hline CMT middle third & 1.34 & 1.33 & 0.94 & 1.99 & 0.22 & 1.34 & 1.33 & 0.93 & 1.73 & 0.17 \\
\hline CMT lower third & 1.18 & 1.15 & 0.68 & 1.83 & 0.26 & 1.19 & 1.18 & 0.76 & 1.83 & 0.23 \\
\hline CMT proximal & 1.42 & 1.43 & 0.97 & 1.89 & 0.21 & 1.43 & 1.44 & 1.09 & 1.79 & 0.18 \\
\hline CMT distal & 1.32 & 1.37 & 0.82 & 1.70 & 0.22 & 1.34 & 1.35 & 0.97 & 1.78 & 0.21 \\
\hline
\end{tabular}

RMT - rested muscle thickness; CMT - contracted muscle thickness; SD - standard deviation.

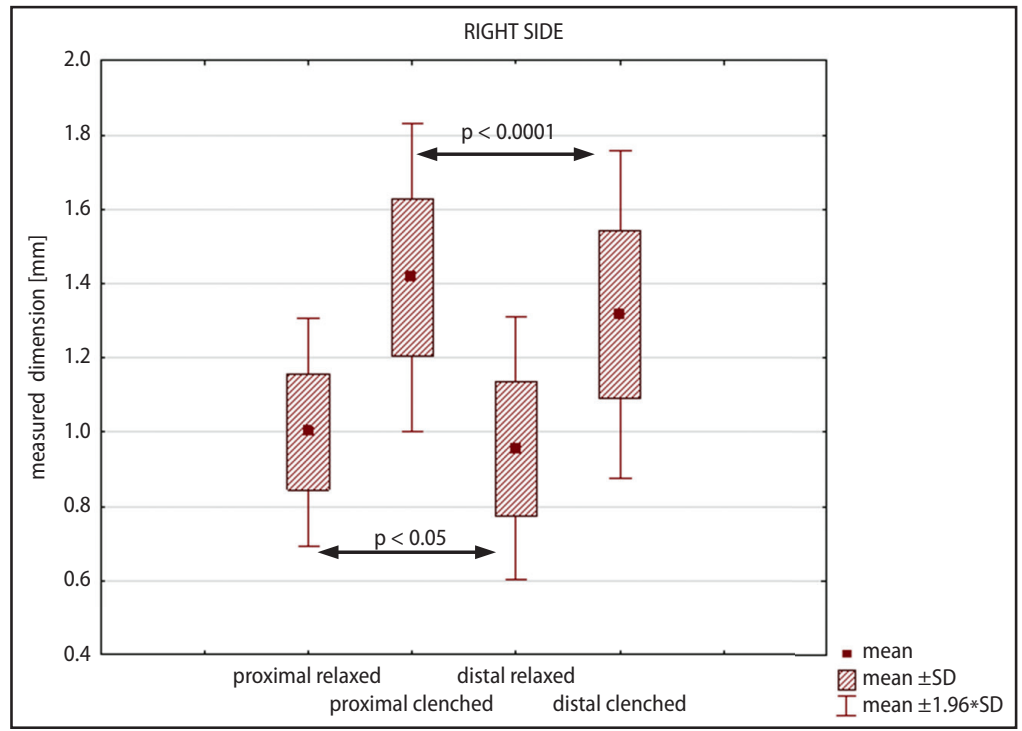

Fig. 6. Statistical imaging of ultrasound measurements at proximal and distal areas of masseter at RMT and CMT records, with the p-level on the right side

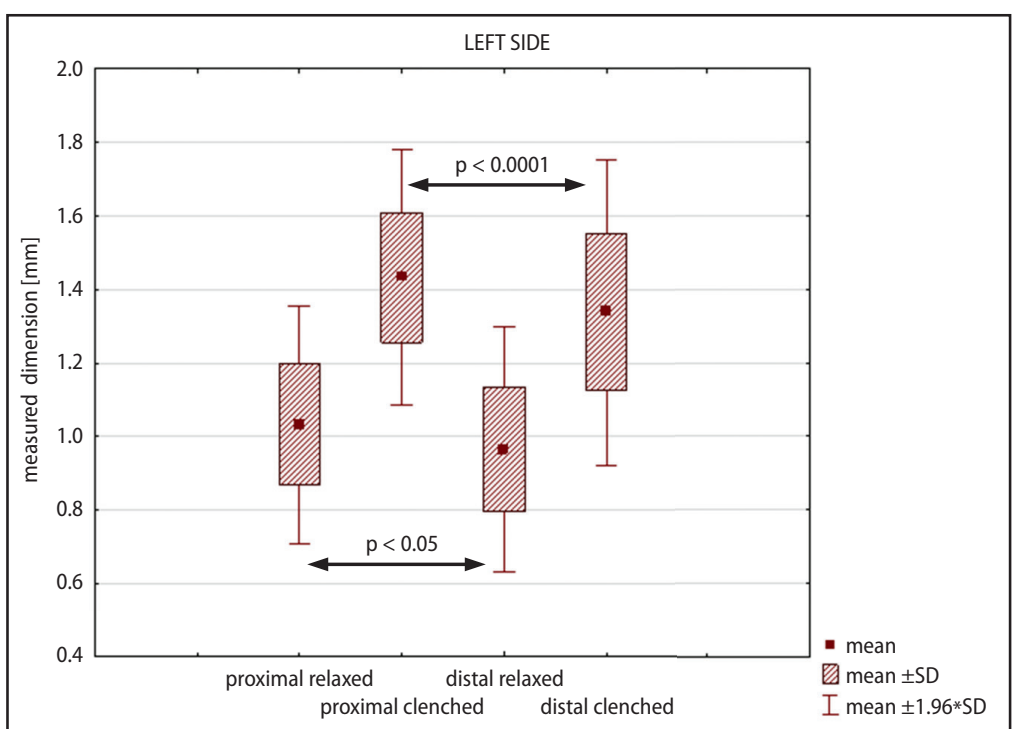

Fig. 7. Statistical imaging of ultrasound measurements at proximal and distal areas of masseter at RMT and CMT records, with the p-level on the left side between masseter muscle thickness measured in the upper and middle areas during relaxation. No statistically significant differences were found between left and right sides or between males and females $(\mathrm{p}>0.05)$. Expected morphological differences were found at ultrasound records. Clinically, the minimum mean masseter muscle thickness was recorded in the lower third of the muscle at rest $(0.74 \pm 0.2 \mathrm{~mm})$ and the maximum mean in the proximal part of contracted muscle $(1.42 \pm 0.2 \mathrm{~mm})$. The recorded masseter muscle thickness measurements are presented in Table 1.

The ultrasound study revealed very high $(\mathrm{p}<0.0001)$ or high $(\mathrm{p}<0.001)$ statistical differences between RMT and CMT in most designated areas. Only RMT measurement differences between upper and middle areas revealed no statistical difference, while the difference of proximal compared to distal region was significant at $\mathrm{p}<0.05$ (Fig. 4-7).

The difference between CMT and RMT was most significant in the lower third of the muscle. The greatest increase in recorded dimensions during contraction was found at the lower (54\%) and anterior (41\%) regions of the muscle. The results revealed that the difference between CMT and RMT results in higher statistical and clinical meaning than a single measurement. This emphasizes the meaning of the FITD calculation, which describes functional status and is not a size-relative diagnosis.

\section{Discussion}

The presented study recorded average results of $9.15 \pm 1.6 \mathrm{~mm}$ and $13.1 \pm 1.9 \mathrm{~mm}$ for RMT and CMT, respectively, with no significant 
differences between the right and the left side or between women and men.

The results are in agreement with 2 previous ultrasound studies, ${ }^{6,25}$ where no dimensional differences in the masseter muscle between women and men were observed. However, other studies showed significantly greater masseter muscle thickness in men than in women in both relaxed and contracted states. ${ }^{11,26}$ In another study, ${ }^{5}$ authors observed that masseter muscle thickness in women during both relaxed and contracted conditions was significantly lesser than in men. They also concluded that masseter muscle thickness during contraction in women was significantly related to body weight and body constitution.

The dimensions of the masseter muscle obtained in the present study are similar to those previously reported. Recorded mean contracted masseter muscle thickness was $15.1 \pm 1.9 \mathrm{~mm}$ in men and $13.0 \pm 1.8 \mathrm{~mm}$ in women. ${ }^{5}$ Another study ${ }^{24}$ reported masseter muscle thickness as $13.3 \pm 1.4 \mathrm{~mm}$ in men and $10.9 \pm 1.3 \mathrm{~mm}$ in women (when relaxed), and $15.5 \pm 1.8 \mathrm{~mm}$ in men and $13.0 \pm 1.2 \mathrm{~mm}$ in women (when contracted). A different study ${ }^{9}$ examined only women and measured masseter muscle thicknesses from $8.83 \mathrm{~mm}$ to $11.08 \mathrm{~mm}$ with the muscle relaxed, and from $9.84 \mathrm{~mm}$ to $12.57 \mathrm{~mm}$ during contraction. An investigation of the thickness during contraction in 252 individuals ${ }^{27}$ revealed an average of $12.3 \pm 2.7 \mathrm{~mm}$.

In the present study, the maximum value of the masseter muscle thickness was recorded in the middle third of the contracted muscle. When both contracted and relaxed, the muscle was thickest in the middle region and thinnest in the lower region. When divided vertically, the proximal region was found to be thicker than the distal one in both contracted and relaxed muscles. Moreover, the greatest difference between RMT and CMT was found in the lower region of the muscle. These findings are in agreement with another study ${ }^{26}$ where the thickest part of the masseter was recorded in the area between the inferior border of the mandible and the virtual line drawn from the angle of the mouth to the ear lobe.

These results encourage consideration of the anatomical and functional organization of the masseter muscle. Two parts of the muscle (superficial and deep) are commonly distinguished, but 3 well-differentiated parts (superficial, intermediate and deep) have been also reported. ${ }^{28} \mathrm{~A}$ different study ${ }^{29}$ showed no evidence for further subdivision of the superficial part into anterior, middle and posterior regions, contrary to an earlier report ${ }^{30}$ where the authors proved that the anterior fibers were considerably longer (35\%) than the posterior fibers. Apparently, a difference in fiber length may be evidence for muscle organization into anterior and posterior parts. This finding was a highly significant result in this study.

Surprisingly, the use of FITD allowed for different results. The analysis of all parameters with the difference between RMT and CMT revealed high statistical significance. The FITD seems to be clinically more objective than regular measurements of muscle dimensions that can present wide differences relative to the size of the subject. The FITD is thought to be independent from muscle thickness and describes functional increases in muscle dimensions. It correlates strongly with the location of the ultrasound measurement. The clinician must be aware of the fact that a single measurement, particularly in relaxed muscle position, may not reveal hypothetical pathology of muscle thickness as strongly as an increase in the difference between RMT and CMT.

The authors proved that the region of examination and the position of the probe influenced the final result of the measurement. The statistically significant differences were observed between series of measurements in different areas. Our results did not allow the best position for examination to be indicated.

Recently, other ultrasound techniques, such as elastography, have been introduced. ${ }^{31}$ This method potentially allows assessment of not only morphological or functional changes in dimensions but also function of the muscle fibers. However, this method is still under investigation for improved diagnosis of TMD and for monitoring the effects of management.

The ultrasound examination is cost-effective, repeatable (unlike electromyography (EMG)), free from contraindications (unlike magnetic resonance imaging (MRI)), and more objective than clinical examination because it does not reflect the patient's self-reported data. The presented protocol emphasizes clinical use, makes it reliable for functional diagnostics, and enables objective examination of muscle strength and muscle bilateral symmetry. We are convinced that the authors' original method of examination standardizes the clinical protocol, and allows objective comparison of the population. The presented FITD calculation allows size-independent data to be recorded and can be easily introduced into clinical examination. Further studies describing the relationship of FITD to clinical disorders seem to be needed in the future.

\section{Limitations}

This method could include age- and weight-related statistics as well as occlusion related morphological relations in masseter US findings.

\section{Conclusions}

The ultrasound study showed that masseter muscle thickness significantly differs depending on the examined area. The authors emphasize the necessity to examine the masseter muscle in specified areas with both coronal and axial projections to achieve objective and repeatable examination. Notable clinical value is assigned to FITD, which is independent from morphological dimensions of the muscle. 


\section{ORCID iDs}

Krzysztof Gawriołek (D) https://orcid.org/0000-0002-2590-2372 Tomasz Klatkiewicz (1) https://orcid.org/0000-0002-4994-7612 Agnieszka Przystańska (D) https://orcid.org/0000-0003-0012-9819 Zofia Maciejewska-Szaniec (D) https://orcid.org/0000-0002-1965-0498 Tomasz Gedrange (D) https://orcid.org/0000-0002-3551-6467 Agata Czajka-Jakubowska (D) https://orcid.org/0000-0002-1692-2910

\section{References}

1. Busato A, Balconi G, Vismara V, Bertelè L, Tonti G, Pedrizzetti G. Strain analysis of masseter muscle by ultrasound. J Biol Regul Homeost Agents. 2015;29(3 Suppl 1):74-81. PMID:26511184

2. Busato A, Balconi G, Vismara V, Bertelè L, Garo G, De Gregorio D. Ultrasound and analysis of the deformation patterns of the masseter muscle: Comparing surgical anatomy, ultrasound and functional anatomy. Oral Implantol (Rome). 2017;9(Suppl 1/2016 to N 4/2016):28-37. doi:10.11138/orl/2016.9.1S.028

3. Serra MD, Duarte Gavião MB, dos Santos Uchôa MN. The use of ultrasound in the investigation of the muscles of mastication. Ultrasound Med Biol. 2008;34(12):1875-1884. doi:10.1016/j.ultrasmedbio.2008. 05.009

4. Dimova-Gabrovska M, Dimitrova D. Ultrasound diagnostic of musculus masseter. Journal of IMAB. 2017;23(2):1611-1615. doi:10.5272/ jimab.2017232.1611

5. Kiliaridis S, Kalebo P. Masseter muscle thickness measured by ultrasonography and its relation to facial morphology. J Dent Res. 1991;70(9): 1262-1265. doi:10.1177/00220345910700090601

6. Prabhu NT, Munshi AK. Measurement of masseter and temporalis mus cle thickness using ultrasonographic technique. J Clin Pediatr Dent. 1994;19(1):41-44. PMID:7865422

7. Reis Durão AP, Morosolli A, Brown J, Jacobs R. Masseter muscle measurement performed by ultrasound: A systematic review. Dentomaxillofac Radiol. 2017;46(6):20170052. doi:10.1259/dmfr.20170052

8. Naser-ud-Din S, Thoirs K, Sampson WJ. Ultrasonography, lateral cephalometry and 3D imaging of the human masseter muscle. Orthod Craniofac Res. 2011;14(1):33-43. doi:10.1111/j.1601-6343.2010.01505.x

9. Bakke M, Tuxen A, Vilmann P, Jensen BR, Vilmann A, Toft M. Ultrasound image of human masseter muscle related to bite force, electromyography, facial morphology, and occlusal factors. Scand J Dent Res. 1992;100(3):164-171. doi:10.1111/j.1600-0722.1992.tb01734.x

10. Rani S, Ravi MS. Masseter muscle thickness in different skeletal morphology: An ultrasonographic study. Indian J Dent Res. 2010;21(3): 402-407. doi:10.4103/0970-9290.70812

11. Tircoveluri S, Singh JR, Rayapudi N, Karra A, Begum M, Challa P. Correlation of masseter muscle thickness and intermolar width: An ultrasonography study. J Int Oral Health. 2013;5(2):28-34. PMID:24155588

12. Aldemir K, Üstüner E, Erdem E, Demiralp AS, Oztuna D. Ultrasound evaluation of masseter muscle changes in stabilization splint treatment of myofascial type painful temporomandibular diseases. Oral Surg Oral Med Oral Pathol Oral Radiol. 2013;116(3):377-383. doi:10. 1016/j.0000.2013.06.011

13. Chakarvarty A, Panat SR, Sangamesh NC, Aggarwal A, Jha PC. Evaluation of masseter muscle hypertrophy in oral submucous fibrosis patients: An ultrasonographic study. J Clin Diagn Res. 2014;8(9):ZC45-ZC47. doi:10.7860/JCDR/2014/8892.4857

14. Kant P, Bhowate RR, Sharda N. Assessment of cross-sectional thickness and activity of masseter, anterior temporalis and orbicularis oris muscles in oral submucous fibrosis patients and healthy controls: An ultrasonography and electromyography study. Dentomaxillofac Radiol. 2014;43(3):20130016. doi:10.1259/dmfr.20130016

15. Bhoyar PS, Godbole SR, Thombare RU, Pakhan AJ. Effect of complete edentulism on masseter muscle thickness and changes after complete denture rehabilitation: An ultrasonographic study. J Investig Clin Dent. 2012;3(1):45-50. doi:10.1111/j.2041-1626.2011.0088.x
16. Müller F, Hernandez M, Grütter L, Aracil-Kessler L, Weingart D, Schimmel M. Masseter muscle thickness, chewing efficiency and bite force in edentulous patients with fixed and removable implant-supported prostheses: A cross-sectional multicenter study. Clin Oral Implants Res. 2012;23(2):144-150. doi:10.1111/j.1600-0501.2011.02213.x

17. Sathasivasubramanian S, Venkatasai PM, Divyambika CV, et al. Masseter muscle thickness in unilateral partial edentulism: An ultrasonographic study. JClin Imaging Sci. 2017;7:44. doi:10.4103/jcis.JCIS_50_17

18. Schimmel M, Loup A, Duvernay E, Gaydarov N, Muller F. The effect of mandibular denture abstention on masseter muscle thickness in a 97-year-old patient: A case report. Int J Prosthodont. 2010;23(5): 418-420. PMID:20859556

19. Trawitzki LV, Dantas RO, Elias-Júnior J, Mello-Filho FV. Masseter muscle thickness three years after surgical correction of class III dentofacial deformity. Arch Oral Biol. 2011;56(8):799-803. doi:10.1016/j.archoral bio.2011.01.012

20. Umeki K, Watanabe $\mathrm{Y}$, Hirano $\mathrm{H}$, et al. The relationship between masseter muscle thickness and appendicular skeletal muscle mass in Japanese community-dwelling elders: A cross-sectional study. Arch Gerontol Geriatr. 2018;78:18-22. doi:10.1016/j.archger.2018.05.014

21. Vasconcelos PB, de Sousa LG, Regalo SC, et al. The influence of maxillary and mandibular osteoporosis on maximal bite force and thickness of masticatory muscles. Acta Odontol Latinoam. 2015;28(1):2227. doi:10.1590/S1852-48342015000100003

22. Klatkiewicz T, Gawriołek K, Pobudek-Radzikowska M, CzajkaJakubowska A. Ultrasonography in the diagnosis of temporomandibular disorders: A meta-analysis. Med Sci Monit. 2018;24:812-817. doi:10.12659/msm.908810

23. Oliveira J, Filho M, Melo T, Lima N, Filho M, Silva H. Evidence of measures of normalcy for thickness of masseter muscle evaluated with ultrasound. Revista CEFAC: actualização científica em fonoaudiologia. 2015;17(1):238-252. https://doi.org/10.1590/1982-021620150514

24. Schiffman E, Ohrbach R, Truelove E, et al; International RDC/TMD Consortium Network; International Association for Dental Research; Orofacial Pain Special Interest Group; International Association for the Study of Pain. Diagnostic Criteria for Temporomandibular Disorders (DC/TMD) for clinical and research applications: Recommendations of the International RDC/TMD Consortium Network and Orofacial Pain Special Interest Group. J Oral Facial Pain Headache. 2014; 28(1):6-27. doi:10.11607/jop.1151

25. Pereira LJ, Gavião MB, Bonjardim LR, Castelo PM, van der Bilt A. Muscle thickness, bite force, and craniofacial dimensions in adolescents with signs and symptoms of temporomandibular dysfunction. Eur J Orthod. 2007;29(1):72-78. doi:10.1093/ejo/cjl055

26. Strini PJ, Strini PJ, de Sousa Barbosa T, Gavião MB. Assessment of thickness and function of masticatory and cervical muscles in adults with and without temporomandibular disorders. Arch Oral Biol. 2013;58(9): 1100-1108. doi:10.1016/j.archoralbio.2013.04.006

27. Xie Y, Zhou J, Li H, Cheng C, Herrler T, Li Q. Classification of masseter hypertrophy for tailored botulinum toxin type A treatment. Plast Reconstr Surg. 2014;134(2):209e-218e. doi:10.1097/PRS.00000 00000000371

28. Gaudy JF, Zouaoui A, Bravetti P, Charrier JL, Guettaf A. Functional organization of the human masseter muscle. Surg Radiol Anat. 2000;22(3-4):181-190. doi:10.1007/s00276-000-0181-5

29. Zwijnenburg AJ, Lobbezoo F, Kroon GW, Naeije M. Mandibular movements in response to electrical stimulation of superficial and deep parts of the human masseter muscle at different jaw positions. Arch Oral Biol. 1999;44(5):395-401. doi:10.1016/s0003-9969(99)00016-3

30. van Eijden TM, Raadsheer MC. Heterogeneity of fiber and sarcomere length in the human masseter muscle. Anat Rec. 1992;232(1):78-84. doi:10.1002/ar.1092320109

31. Olchowy A, Wieckiewicz M, Winocur E, et al. Great potential of ultrasound elastography for the assessment of the masseter muscle in patients with temporomandibular disorders: A systematic review. Dentomaxillofac Radiol. 2020;49(8):20200024. doi:10.1259/dmfr.20200024 